\title{
Effect of Picosecond Laser Based Modifications of Amorphous Carbon Coatings on Lubricant-free Tribological Systems
}

\author{
Tom Häfner ${ }^{* 1,2}$, Johannes Heberle ${ }^{* 1,2}$, Hubert Hautmann ${ }^{* 1}$, Rong Zhao*3, Jennifer Tenner ${ }^{* 4}$, Stephan Tremmel ${ }^{* 3}$, \\ Marion Merklein ${ }^{* 2,4}$, Michael Schmidt ${ }^{* 1,2}$ \\ ${ }^{* 1}$ Institute of Photonic Technologies, Friedrich-Alexander-Universität Erlangen-Nürnberg, \\ Konrad-Zuse-Str. 3/5, 91052 Erlangen, Germany \\ E-mail: tom.haefner@fau.de \\ *2 Erlangen Graduate School in Advanced Optical Technologies (SAOT), Friedrich-Alexander- \\ Universität Erlangen-Nürnberg, Paul-Gordan-Str. 6, 90152 Erlangen, Germany \\ ${ }^{* 3}$ Institute of Engineering Design, Friedrich-Alexander-Universität Erlangen-Nürnberg, \\ Martensstr. 9, 91058 Erlangen, Germany \\ ${ }^{* 4}$ Institute of Manufacturing Technology, Friedrich-Alexander-Universität Erlangen-Nürnberg, \\ Egerlandstr. 13, 91058 Erlangen, Germany
}

\begin{abstract}
Motivated by the efficient usage of resources, friction and wear between tool and workpiece are increased by the abandonment of lubricants in metal forming processes. To substitute the functions of lubricants and to control the material flow in dry deep drawing processes surface modifications such as tetrahedral amorphous carbon (ta-C) coatings are essential. Local tailored tribological conditions can be realized by selective ultrashort laser based treatment of ta-C coatings. The effects of picosecond laser processing on the surface morphology, chemical and mechanical properties of the coatings are presented. The analysis of wavelength dependent threshold fluences for graphitization and ablation and the quality of ta-C coatings reveals that homogenous heat treatment and partial material removal can be achieved by processing with $\lambda=355 \mathrm{~nm}$. The proper adjustment of fluence and pulse overlap enables heat treatment of the coating surface due to heat accumulation. The induced structural changes of the treated coating decrease elastic indentation modulus and affect the friction coefficient which is evaluated in flat strip drawing tests. Beyond that, laser structuring of the coated surfaces leads to either friction decrease or increase depending on the degree of coverage and the orientation of the microstructures.

DOI: 10.2961/jlmn.2017.02.0015
\end{abstract}

Keywords: picosecond laser, ablation, heat treatment, tetrahedral amorphous carbon, coating, lubricant-free, tribology

\section{Introduction}

Ecological and economical aspects mainly motivate dry sheet metal forming [1]. Lubricants which are conventionally applied in forming operations are environmentally harmful and have to be removed by cleaning after forming and prior to additional production steps. Thus, these lubricants have to be omitted to provide efficient usage of resources. The abandonment of lubricants leads to increased friction and distinctive wear due to more intensive interaction between tool and workpiece [2]. One approach to face the resulting challenges in the deep drawing process is represented by the concept of a tailored tool with locally adapted friction conditions. For this purpose, different types of surface modifications have to be investigated and developed to provide tailored tribological systems. Different friction conditions in lubricant-free tribological systems should allow the control of material flow in deep drawing processes. One approach for the essential friction reduction and the substitution of the functions of conventional lubricants is represented by the application of amorphous carbon coatings. Amorphous carbon coatings are advanta- geous to decrease friction and to increase wear resistance. In lubricant-free tribological systems these coatings are advantageous due to its chemical inertness and high hardness [3]. Amorphous carbon coatings - also called diamond-like carbon (DLC) - are distinguished by their hydrogen-content as well as their ratio of $\mathrm{sp}^{2}$ (graphite) and $\mathrm{sp}^{3}$ (diamond) phases according to the ternary diagram [4].

In the framework of this paper, tetrahedral amorphous carbon (ta-C) coatings are applied to reduce adhesion tendency and thus, friction in contact with the tested sheet materials - the mild deep drawing steel DC04 and the aluminum alloy AA5182. Post-deposition laser processing of the coating surface provides an additional possibility for the local acceleration and deceleration of the material flow due to contact zones with low and high friction. The adaption of surface topography on the macroscopic scale by laser based material ablation of the coating represents one approach of local adaption of friction conditions [5]. Such microstructures can change the real contact area and provide capacity to collect wear particles from the contact zone. The influence of structured ta- $C$ coatings on dry tribological systems has already been studied by ROCH ET AL. [6]. However, they investigated nanosecond laser based 
processing of the coatings which induces phase changes of the coating in the comparatively large heat affected zones (HAZ). Thus, the influence of the topography on the tribological behavior cannot be separately distinguished from the chemical modifications which are induced due to heating. Although, the influence of nanosecond laser based heat treatment on the structural properties was investigated experimentally and numerically [7], the effect on the tribological conditions according to the conditions in dry deep drawing is not known. Furthermore, predominant beammatter interaction mechanisms in picosecond laser processing can differ from mechanisms which cause material heating and ablation by nanosecond pulses.

Therefore, this paper deals with the influence of modifications of ta-C coatings which were generated by picosecond (ps) laser based processing. Ultrashort pulsed laser processing of amorphous carbon coatings enables tailored surface modification of the coating properties within one processing step by structuring as well as local heat treatment due to heat accumulation with a single laser source. Applying well-chosen processing parameters material ablation with minimal HAZ as well as local heat treatment are investigated.

First, appropriate processing parameters including the wavelength are studied for homogeneous processing of the ta-C coating. Regarding ablation of the ta- $\mathrm{C}$ coating structure properties are evaluated to allow precise and welldefined microstructuring. Varying the degree of coverage and the orientation of microstructures the possible range for adjustment of the friction coefficient is evaluated. The result of the tribological investigations will confirm the hypothesis, that friction can be either decreased or increased due to the contacting asperities in the tribological system. For this purpose, $6 \mu \mathrm{m}$ deep structures should provide sufficient volume to enable collection of wear particles in contact with DC04. In contrast, flat structures with a depth of $0.6 \mu \mathrm{m}$ are investigated in contact with AA5182 to provide workpiece surfaces which are completely covered with carbon because of the high adhesion tendency of aluminum and the limited thickness of the tested ta-C coatings.

For ps laser based heat treatment, the influence of the degree of $\mathrm{sp}^{2}$ hybridization of ta-C coatings on the tribological behavior is examined to enable the adjustment of the friction coefficient without topographical modifications. Furthermore, the influence of the interaction between coating properties and tribological behavior might be better understood as a result of these investigations. To realize reliable and reproducible post-deposition adjustment of the chemical and mechanical properties a wide-spread process window for heat treatment is determined. The temperature field in the ta-C layer during ps laser based heat treatment is numerically simulated to justify the dependency of dimensions of graphitized regions on the processing parameters. Furthermore, the calculated temperatures are used to correlate the results of RAMAN spectroscopy and micro hardness measurements with the laser based energy input. Finally, the tribological impact of the generated chemical and mechanical layer modifications is evaluated by means of determining the friction coefficient in dry flat strip drawing tests and characterizing the surfaces.

\section{Experimental setup and methods}

\subsection{Substrate and coating materials}

In this paper, the cold working steel 1.2379 (X155CrVMo12) with a hardness of 60 HRC was used as substrate material. Prior to coating deposition, the substrate surfaces were finished by a combined lapping and rough polishing process resulting in a roughness characterized by a reduced peak height of $S p k=0.09 \pm 0.02 \mu \mathrm{m}$ (scope of measurements $n=3$ ). On the tool steel surface a tetrahedral hydrogen free amorphous carbon coating system (ta-C) was deposited. The coating system consists of chrome (Cr) adhesive layer of about $0.6 \mu \mathrm{m}$ which was generated by sputtering and the ta-C functional layer which has a thickness of about $0.6 \mu \mathrm{m}$. The ta-C layer was generated by laser cathodic vacuum arc process which was initiated by pulsed laser ablation of a graphite target [7]. A magnetic field removed macro particles during coating deposition to reduce the coating roughness. The coated samples were mechanically brushed to realize the surface roughness Spk $=0.04 \pm 0.02 \mu \mathrm{m}(n=3)$.

To investigate the tribological behavior of modified ta-C coatings the zinc coated mild deep drawing steel DC04 and the aluminum alloy AA5182 with an initial thickness of $1 \mathrm{~mm}$ were applied as sheet materials. The surfaces of both sheets have an electrical discharge texture (EDT).

\subsection{Picosecond laser processing}

Prior to laser processing the samples were ultrasonically cleaned for $3 \mathrm{~min}$ in isopropanol to remove any residues. For laser processing the mode-locked $\mathrm{Nd}: \mathrm{YVO}_{4}$ laser system Fuego (Time-Bandwidth Products) with the pulse duration $\tau_{P}=10$ ps (FHWM) was applied. The specifications of this systems for the wavelengths $\lambda_{1}=1064 \mathrm{~nm}$ and $\lambda_{2}=355 \mathrm{~nm}$ are listed in Table 1. Depending on the applied wavelength the relative movement between laser beam and sample surface was either realized by the galvanometer scanner hurryScan 14 II (Scanlab AG) for $\lambda_{1}=1064 \mathrm{~nm}$ or by the movement of the sample by means of linear $x-y$ stages ANT150-L (Aerotech) for $\lambda_{2}=355 \mathrm{~nm}$. Each experiment was carried out at room temperature and at atmospheric pressure. After laser machining the laser treated surfaces are cleaned in an ultrasonic bath with isopropanol.

Table 1 Specification of setup and laser system Fuego

\begin{tabular}{lcc}
\hline & $\lambda_{1}=1064 \mathrm{~nm}$ & $\lambda_{2}=355 \mathrm{~nm}$ \\
\hline Pulse frequency $\mathrm{f}_{\mathrm{P}}(\mathrm{kHz})$ & Single pulse $-8,200$ \\
Max. pulse energy $\mathrm{E}_{\mathrm{P}}(\mu \mathrm{J})$ & 150 & 55 \\
Focal length $\mathrm{f}(\mathrm{mm})$ & 160 & 150 \\
Radius at beam waist $\mathrm{w}_{0}(\mu \mathrm{m})$ & 15.5 & 15.5 \\
\hline
\end{tabular}

For the tribological characterization of structured ta-C coated surfaces, microstructures with six different geometries were generated. The rectangular (R20d, R35d, R50d) structures (length $500 \mu \mathrm{m}$ and width $200 \mu \mathrm{m}$ ) with different degree of coverage (20\%; $35 \%$; $50 \%$ ) and $6 \mu \mathrm{m}$ depth (d) are generated by both parameter sets A.1 and A.2 (Table 2). First, A.1 was applied to locally remove the ta-C coating and was followed by set A.2 for the ablation of the steel substrate. The flat rectangular (R35f) and line-shaped 
(L35f) structures with a depth of $0.6 \mu \mathrm{m}$ and $35 \%$ degree of coverage were generated by applying only A.1. The lineshaped structures L35f* (width $200 \mu \mathrm{m}$ ) with a depth of $0.6 \mu \mathrm{m}$ were processed by set $\mathrm{B}$ and have a bottom still covered with carbon.

Table 2 Laser processing parameters for microstructuring

\begin{tabular}{lccccc}
\hline Set & $\begin{array}{c}\text { Wave- } \\
\text { length } \lambda \\
(\mathrm{nm})\end{array}$ & $\begin{array}{c}\text { Fluence F } \\
\left(\mathrm{J} / \mathrm{cm}^{2}\right)\end{array}$ & $\begin{array}{c}\text { Pitch } \\
\text { distance } \mathrm{p}_{\mathrm{x}} \\
(\mu \mathrm{m})\end{array}$ & $\begin{array}{c}\text { Hatch } \\
\text { distance } \mathrm{p}_{\mathrm{y}} \\
(\mu \mathrm{m})\end{array}$ & $\begin{array}{c}\text { No. of } \\
\text { scans S }\end{array}$ \\
\hline A.1 & 1064 & 0.3 & 7 & 10 & 1 \\
A.2 & 1064 & 1.3 & 2 & 10 & 5 \\
B & 355 & 0.6 & 5 & 8 & 2 \\
\hline
\end{tabular}

\subsection{Method and setup for tribological characterization}

The flat strip drawing test was applied to characterize the tribological behavior of the ta- $\mathrm{C}$ coated and modified sample surfaces. The tribological conditions in the flange area of the blank holder in deep drawing are modeled by this setup which is schematically shown in Fig. 1. A sheet metal strip is located between an upper and a lower friction jaw. The normal force $F_{N}$ is applied by the lower friction jaw which is moving upwards. The strip is clamped on one side and is drawn through the jaws with the drawing force $F_{D}$ which equals the sum of upper and lower friction forces $F_{F U}$ and $F_{F L}$. The friction coefficient $\mu$ is determined between the length of 100 to $170 \mathrm{~mm}$ by applying the Coulomb friction law.

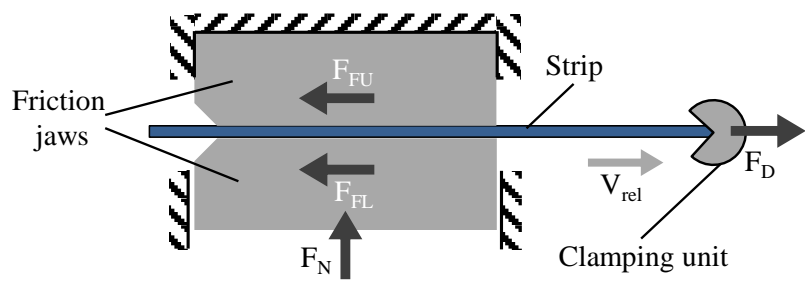

Fig. 1 Schematic setup of flat strip drawing test

The strips were cleaned in an acetone bath prior to the drawing tests to remove basic lubrication and soil. The removal of the substances was proved by an infrared sensor. The relative velocity $v_{\text {rel }}$ was set to $100 \mathrm{~mm} / \mathrm{s}$ which is a typical velocity for deep drawing. The contact pressure for DC04 was set to $p_{N}=4.5 \mathrm{MPa}$ while for AA5182 a lower pressure $p_{N}=1.5 \mathrm{MPa}$ was chosen due to its lower tensile strength. The experiments were repeated three times and were performed at room temperature and ambient air with relative humidity of $29 \pm 12 \%$.

\subsection{Characterization of the sample surface}

Before and after laser processing as well as after flat strip drawing tests the sample surface were topographically characterized by measurements with a confocal laser scanning microscope (LSM) LEXT (Olympus) and a confocal white light scanning microscope $\mu$ Surf (NanoFocus). The surface roughness parameters were optically measured according to ISO 25178 with the LSM [9]. The scanning electron microscope (SEM) Merlin Gemini II (Zeiss) was used to investigate the morphology on the micro- and nanometer scale of the untreated and laser irradiated surfaces.
Micro RAMAN spectroscopy (alpha 300RA, WITec) was used to analyze the chemical properties of the (modified) ta-C layers. Therefore, the chemical bond configuration was investigated by recording the inelastic scattering of the laser excitation wavelength $\lambda_{\text {Raman }}=532 \mathrm{~nm}$. RAMAN analysis was applied in the range of 800 to $2500 \mathrm{~cm}^{-1}$ where ta-C coatings have their characteristic peaks [10]. Focusing the applied laser power of $10 \mathrm{~mW}$ by a $10 \mathrm{x}$ or 20x objective on the ta-C coated surface undesired modifications of the coating structure were avoided [11]. A linear fit for the background as well as a Breit-Wigner-Fano (BWF) and a Lorentzian model were used to decompose the recorded spectra and calculate the intensity for the $G$ and D peaks [10]. Micro hardness was determined by Vickers indentation (Fischerscope H100VP, Fischer) with indentation force of $10 \mathrm{mN}$ in $10 \mathrm{~s}$ according to DIN EN ISO $14577-1$ at 10 points for each modified surface [12].

\subsection{Simulation model for ps laser based heat treatment}

A solid heat transfer model was built up in COMSOL (Comsol Multiphysics) to evaluate the influence of heat input on dimensions and degree of $\mathrm{sp}^{2}$ hybridization of graphitized regions. This FEM model was applied to calculate the temperature profiles as a function of depth $z$, radius $y$ and time $t$. The axisymmetric model consists of four layers with the following thicknesses: steel substrate $(100 \mu \mathrm{m})$, the chrome adhesive layer $(0.6 \mu \mathrm{m})$, ta-C layer $(0.6 \mu \mathrm{m})$ and air $(300 \mu \mathrm{m})$. Air temperature is defined to $20^{\circ} \mathrm{C}$. The spot radius in the simulation model was defined to $w_{0}=15 \mu \mathrm{m}$ unless specified differently. The governing equation (1) of the model includes energy conservation:

$$
\rho c_{P}(T) \frac{\partial T(r, z, t)}{\partial t}+\rho c_{P}(T) \cdot \nabla T-k \cdot \nabla T=Q
$$

where $\rho$ is the density, $c_{P}(T)$ the heat capacity at constant pressure and $\kappa$ the thermal conductivity. The heat source term $\mathrm{Q}$ is defined by eq. (2):

$$
Q=\frac{2 \cdot E_{P}}{\pi \cdot w_{0}{ }^{2}} \cdot e^{\frac{-2 r^{2}}{w_{0}{ }^{2}}} \frac{2}{\sqrt{2 \pi} \cdot \tau_{P}}(1-R) \cdot \alpha \cdot e^{\alpha z}
$$

with pulse energy $E_{P}$, reflectivity $R$ and absorption coefficient $\alpha$ as well as radius $r$ and depth $z$. The optical properties of the ta-C layer were calculated from a polynomial fit derived by the dependency of the complex refractive index on the ratio of $\mathrm{sp}^{3}$ (diamond) phases. TAY ET AL. [13], ROCH ET AL. [6] and ARAKAWA ET AL. [14] published values of the complex refractive index for $\mathrm{sp}^{3}$-phase contents between $20 \%$ and $85 \%$. The $\mathrm{sp}^{3}$-ratio was assumed to be $80 \%$ for the ta-C layers which were processed in this paper (Table 3).

Table 3 Optical and thermal properties of the ta-C coating

\begin{tabular}{lc}
\hline & $\lambda_{2}=355 \mathrm{~nm}$ \\
\hline Refractive index $\mathrm{n}$ & 3.26 \\
Extinction coefficient $\mathrm{k}$ & 0.31 \\
Absorption coefficient $\alpha\left(\mathrm{cm}^{-1}\right)$ & $10.880 \cdot 10^{4}$ \\
Reflectivity R & 0.28 \\
\hline Emissivity E (of carbon) $\left(25^{\circ} \mathrm{C}\right)$ & 0.81 \\
Density $\delta\left(\mathrm{g} \cdot \mathrm{cm}^{-3}\right)[17]$ & 3.0 \\
Thermal conductivity $\kappa\left(\mathrm{W} \cdot \mathrm{m}^{-1} \cdot \mathrm{K}^{-1}\right)[17]$ & 3.5 \\
Debye temperature $\Theta_{\mathrm{D}}(\mathrm{K})[17]$ & 1217 \\
\hline
\end{tabular}


This assumption is justified by the results of indentation measurements [15] and the $I_{D} / I_{G}$-ratio received by RAMAN spectroscopy which is in the range of similar ta-C layers given in literature [16]. The volumetric heat capacity $C_{P}(T)$ of ta-C is calculated according to the well-known DEBYE model [18] given by eq. (3):

$$
c_{P}(T)=\left[9 \cdot N_{A} \cdot k_{B} \cdot\left(\frac{T}{\theta_{D}}\right)^{3} \int_{0}^{\frac{\theta_{D}}{T}} \frac{x^{4} \cdot e^{x}}{\left(e^{x}-1\right)^{2}} d x\right]\left(\frac{\rho}{M}\right)
$$

where $N_{A}$ and $k_{B}$ are the AvOGADRo and the BoLtzMANN constants, respectively. The molar mass of carbon $M=12 \mathrm{~g} / \mathrm{mol}$ was selected. The thermal properties of air, chrome and tool steel derived from Ref. [19] and [20] are listed in Table 4.

Table 4 Properties of air, chrome (Cr) and steel 1.2379

\begin{tabular}{lcccc}
\hline & & Air & $\mathrm{Cr}$ & 1.2379 \\
\hline $\begin{array}{l}\text { Density } \delta \\
\left(\mathrm{g} \cdot \mathrm{cm}^{-3}\right)\end{array}$ & $\left(\mathrm{T}=20^{\circ} \mathrm{C}\right)$ & 1.189 & 7.15 & 7.70 \\
\hline Heat conductivity $\kappa$ & $\left(\mathrm{T}=200^{\circ} \mathrm{C}\right)$ & 0.736 & 7.15 & 7.65 \\
$\left(\mathrm{~W} \cdot \mathrm{m}^{-1} \cdot \mathrm{K}^{-1}\right)$ & $\left(\mathrm{T}=200^{\circ} \mathrm{C}\right)$ & 0.026 & 94.0 & 16.7 \\
\hline Heat capacity $\mathrm{C}_{\mathrm{P}}$ & $\left(\mathrm{T}=20^{\circ} \mathrm{C}\right)$ & 1006 & 450 & 470 \\
$\left(\mathrm{~J} \cdot \mathrm{kg}^{-1} \cdot \mathrm{K}^{-1}\right)$ & $\left(\mathrm{T}=200^{\circ} \mathrm{C}\right)$ & 1025 & 509 & 530 \\
\hline
\end{tabular}

\section{Results and discussion}

The aforementioned methods have been used to generate well-defined local chemical and topographical modifications of ta-C coatings by picosecond laser processing. Based on the detailed characterization of the modified surfaces the treated samples have been tested in strip drawing tests to understand the interactions in lubricant-free tribological systems.

\subsection{Threshold fluences for modification of ta-C coating}

In a first step during the experimental investigations on picosecond laser based processing of ta-C coatings, the threshold fluences for graphitization and ablation have been evaluated for wavelength $\lambda_{1}=1064 \mathrm{~nm}$ and $\lambda_{2}=355 \mathrm{~nm}$ to realize a tailored energy input (Fig. 2a). Therefore, the widths $B$ of line-shaped structures (exemplarily shown in Fig. 2b) were measured by the zero-damagemethod [21]. The applied effective pulse number $N_{x}$ is calculated according to the first fraction of eq. (4) which defines the effective pulse number $N_{x y}$ for areal laser processing [22]:

$$
N_{x y}=S \cdot N_{x} \cdot N_{y}=\frac{S \cdot 1.25 \cdot w_{0}}{p_{x}} \cdot \frac{1.25 \cdot w_{0}}{p_{y}}
$$

where $S$ is the number of scans, $p_{x}$ the pulse-to-pulse distance in scanning direction (pitch) and $p_{y}$ the line distance perpendicular to the scanning direction (hatch). The graphitization threshold represents the lower limit whereas the ablation threshold defines the upper limit for heat treatment.

For the applied pulse frequency of $1 \mathrm{kHz}$ both thresholds are lower at $1064 \mathrm{~nm}$ than at $355 \mathrm{~nm}$. This observation can be explained by different beam-matter interaction mechanisms at both wavelengths. The energy input at wavelength $\lambda_{2}$ occurs due to linear absorption as the bandgap of comparable ta-C coatings with an $\mathrm{sp}^{3}$ fraction of $80 \%$ is $E_{G}=2.6 \mathrm{eV}$ which corresponds to a wavelength
$\lambda_{G}=477 \mathrm{~nm}$ [13]. The energy for breaking a covalent C-C bond ( $\sigma$ bond) is about $3.61 \mathrm{eV}$ and thus, slightly higher than the energy of a single photon $E_{p h}=3.49 \mathrm{eV}$ at $\lambda_{2}=355 \mathrm{~nm}$. Therefore, linear absorption should be the predominant mechanism of ta-C coating modification. Nevertheless, chemical bond breaking can be induced by the application of high fluences or intensities, respectively, due to multi-photon absorption at this wavelength.

At wavelength $\lambda_{1}$ the ta-C layer is transparent having an absorption coefficient significantly lower than $1 \mathrm{~cm}^{-1}$ and an absorption length $l_{\alpha}>>10 \mu \mathrm{m}$, respectively. Thus, energy absorption within the ta-C layer can mainly occur only via multi-photon absorption. Intensities, which are transmitted by the ta-C layer and absorbed by the chrome layer, heat the chrome layer and can induce either graphitization or confined ablation of the upper ta-C layer at a high intensity. Accordingly, it is assumed that graphitization of the ta-C layer occurs only from its backside via heat conduction. Thus, for defined adjustment of $\mathrm{sp}^{2}$ phase content a non-linear absorption process is disadvantageous.
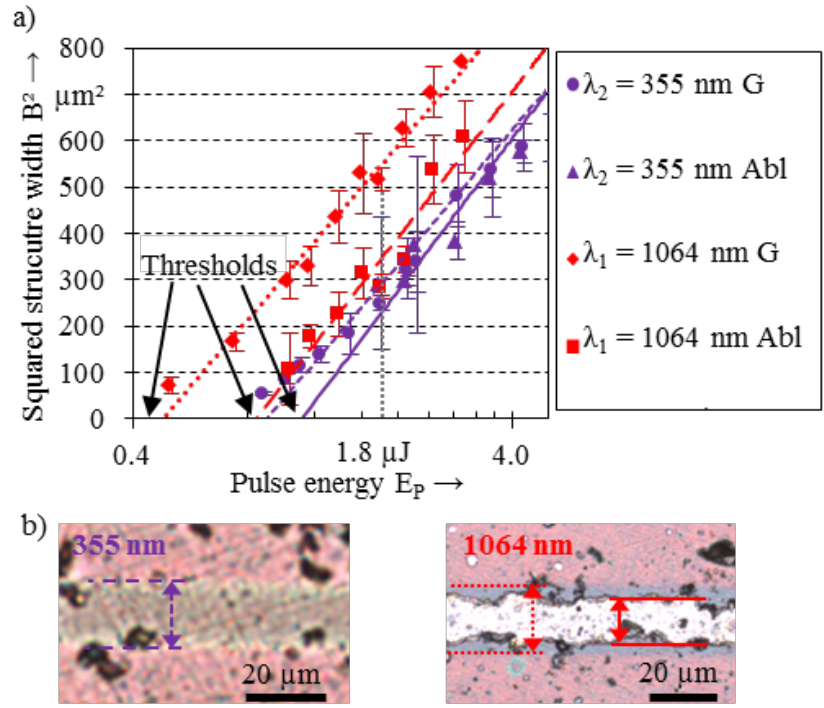

Fig. 2 a) Graphitization (G) and ablation (Abl) thresholds and b) examples of line-shaped structures at $E_{P}=1.8 \mu \mathrm{J}$ $\left(f=1 \mathrm{kHz}, w_{0} \approx 15 \mu \mathrm{m}, p_{x}=4 \mu \mathrm{m}, S=2 ; n=3\right)$

Furthermore, a varying thickness or impurities due to coating deposition increase the risk of undesired stochastic ta-C layer ablation due to significantly higher absorption and spalling, consequently. Spalling represents the mechanism which induces the removal of the whole ta-C layer. The laser intensity is absorbed in the chrome layer inducing ultrafast heating and strong pressure growth in the interface between both layers. In the region where this pressure exceeds the shear strength of the ta-C layer this top layer is removed by confined ablation. The described mechanism can be proved by analyzing the geometry of ablation craters generated by excitation with $\lambda_{1}=1064 \mathrm{~nm}$. Steep edges of the circular crater which was generated by single laser pulse (Fig. 3a) and, as can be seen in Fig. 3a, remaining flakes on the right side of the crater indicate this mechanism. The exposed metallic bottom of the crater and the line-shaped structure (Fig. 3b) indicate spalling of the whole layer, too. The removal of the ta-C layer due to confined ablation enables an efficient structuring process but it is not possible to generate microstructures with a structure 
bottom which is still covered by ta-C coating. Furthermore, homogeneous and reliable surface heat treatment of the top coating layer is hampered due to the risk of ablation induced by inhomogeneities of the ta-C coating. For these reasons, surface processing with $\lambda_{2}=355 \mathrm{~nm}$ is investigated in detail.
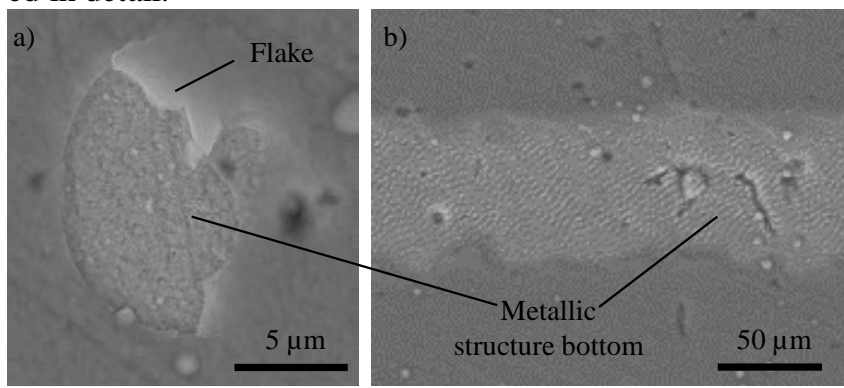

Fig. 3 Edges of a) crater and b) line-shaped structures generated by ablation with $\lambda_{1}=1064 \mathrm{~nm}\left(f_{P}=1 \mathrm{kHz}, w_{0} \approx 15 \mu \mathrm{m}\right.$, $\left.\left.F=0.31 \mathrm{~J} / \mathrm{cm}^{2}, S=1 ; \mathrm{b}\right) p_{x}=4 \mu \mathrm{m}\right)$

\subsection{Picosecond laser based heat treatment}

Efficient and well-defined adjustment of $\mathrm{sp}^{2}$ phase content requires a heat treatment process with high productivity and a process window for graphitization of sufficient width. Therefore, the influence of effective pulse number $N_{x}$, pitch $p_{x}$ and pulse frequency $f_{P}$ on graphitization and ablation threshold was determined for line-shaped structures. A minimal graphitization threshold $F_{t h, G}$ should indicate maximum processing temperature applicable for graphitization and thus, is preferred. Additionally, the maximum temperature in steady state is numerically calculated for different processing parameters by the thermal model. Here we define: The steady state will be achieved, if the peak surface temperature increases from pulse to pulse by less than $0.1 \%$ of the absolute temperature. It is assumed that a higher maximum temperature enables targeted and distinctive heat treatment.

A higher effective pulse number significantly decreases both thresholds while the width of the process window for heat treatment in terms of an applicable fluence range remains nearly constant (Fig. 4). Accordingly, $N_{x}>132$ should be beneficial regarding high maximum temperature. Compared to this, the pitch only marginally affects $F_{t h, G}$ whereby $p_{x}<0.45 \mu \mathrm{m}$ is preferred. In contrast to this, the pulse frequency does not affect the graphitization threshold within the standard deviation which might be due to the high heat conduction of the ta-C coating compared to hydrogenous coatings [17]. As a result, the effective pulse number and the pitch affect more significantly graphitization and ablation threshold than the temporal distance of the laser pulses. Thus, incubation seems to be the most important multi-pulse effect regarding energy input. Phase transformations with increasing $\mathrm{sp}^{2}$ (graphite) hybridization occur due to the high temperature at the ta-C surface which is already induced by the first laser pulse (Fig. 5a). Material properties such as energy penetration depth and heat conductivity dramatically decrease due to graphitization, both leading the energy of subsequent pulses to be distributed within a smaller volume than it occurs for the initial nontreated ta-C coating.

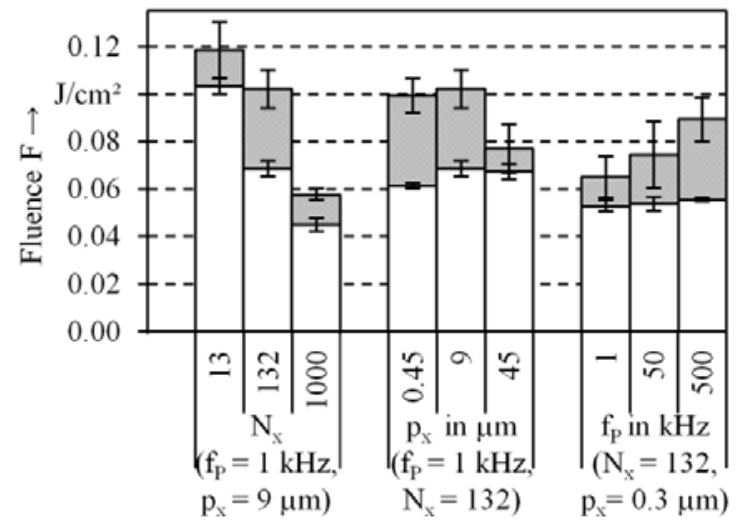

Fig. 4 Threshold for graphitization (lower) and ablation (upper) and shaded process window for heat treatment depending on the parameters $N_{x}, p_{x}$ and $f_{P}$ for line-shaped structures $\left(\lambda_{2}=355 \mathrm{~nm}, w=47.5 \mu \mathrm{m} ; n=3\right)$

Additionally, the peak surface temperatures increase with higher pulse frequency $f_{P}$ (Fig. 5b). For different pulse energies the peak temperatures at $500 \mathrm{kHz}$ exceed the temperatures at $1 \mathrm{kHz}$ up to $90 \mathrm{~K}$ which enables the desired wider process window for heat treatment. Heat accumulation increases with higher $f_{P}$, too. Thus, the maximum width of the process window for heat treatment can be achieved at high $f_{P}$ benefitting from heat accumulation. For $f_{P}=500 \mathrm{kHz}$ the steady state of the peak surface temperature is reached after more than 83 laser pulses depending on the pulse energy. For $f_{P}=1 \mathrm{kHz}$ the change of the peak surface temperature induced by the second laser pulse is already lower. Consequently, heat treatment of ta- $\mathrm{C}$ coatings does not benefit from heat accumulation for pulse frequencies in the single kHz-range. Additionally, productivity of laser processing benefits of higher pulse frequencies due to higher speeds of beam deflection and sample movement, respectively.
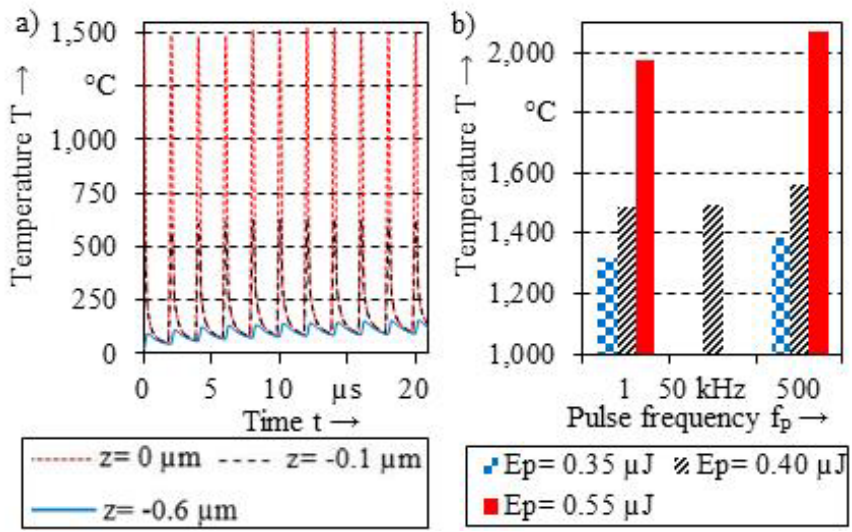

Fig. 5 a) Temperature profiles at the surface and at certain depths $z$ (center of laser pulse $r=0 \mu \mathrm{m}, f_{P}=500 \mathrm{kHz}$ ), b) peak surface temperature after $N=125$ laser pulses depending on pulse energy $E_{P}$ and pulse frequency $f_{P}$

\subsection{Effect on chemical and mechanical properties}

As a result of chapter $3.2, f_{P}=500 \mathrm{kHz}$, spot radius $w=47.5 \mu \mathrm{m}$, pitch $p_{x} \approx 0.3 \mu \mathrm{m}$, effective pulse number $N_{x y} \approx 1000$ and different fluences were applied for areal heat treatment to investigate the influence of energy input on the mechanical and chemical properties. The analysis of the surface topography by LSM measurements did not re- 
veal any modification of the surface roughness due to heat treatment [2]. Micro hardness measurements reveal constant Vickers hardness and indentation modulus for low fluences. Above a certain threshold fluence both mechanical parameters abruptly begin to drop (Fig. 6). Additional increase of the fluence leads only to marginal decrease as hardness and indentation converge to the properties of the steel substrate with its Vickers hardness $1,080 \pm 102$ HV0.001 [23]. Consequently, applying $F>55 \mathrm{~mJ} / \mathrm{cm}^{2}$ leads to mechanical parameters which are significantly lower than the non-treated ta-C reference. The mechanical properties decrease with increasing fluence due to structural changes of the treated coating.

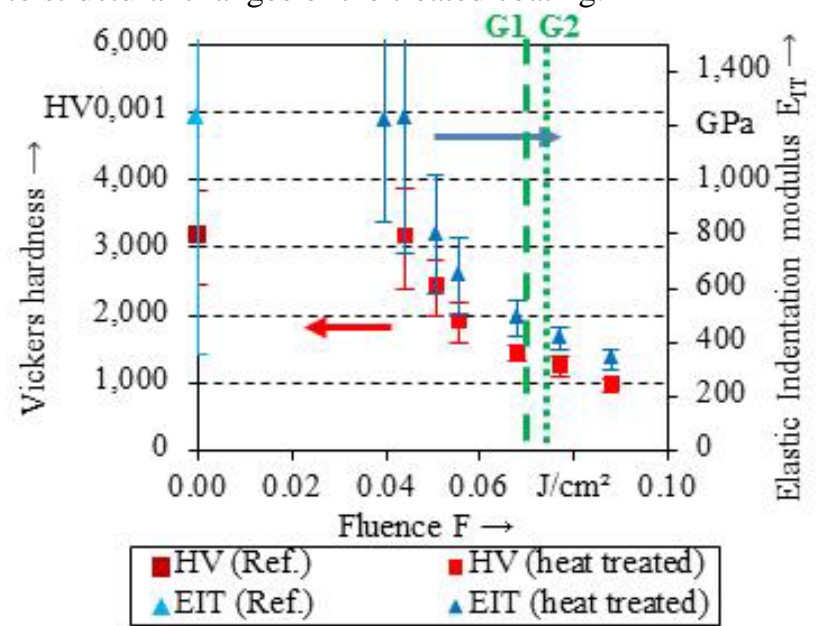

Fig. 6 Vickers hardness $H V$ and elastic indentation modulus $E_{I T}$ depending on the fluence $\left(f_{P}=500 \mathrm{kHz}, w=47.5 \mu \mathrm{m}\right.$, $\left.p_{x}=0.3 \mu \mathrm{m}, p_{y}=15 \mu \mathrm{m}, S=1 ; n=2\right)$. Fluences G1 and G2 were applied to realize different degrees of $\mathrm{sp}^{2}$ hybridization for tribological investigations in chapter 3.5.

The analysis by RAMAN spectroscopy reveals variations of the $I_{D} / I_{G}$-ratio and the position of the $G$ peak depending on the fluence (Fig. 7), which is induced by oscillations of the aliphatically arranged $\mathrm{sp}^{2}$ hybridized phases and thus, represents predominant $\mathrm{sp}^{3}$ (diamond) phases [24]. The spectrum of the reference exhibits one broad peak resulting from the D- (graphite phase) and G-peak (diamond phase). The $I_{D} / I_{G}$-ratio of the reference is about zero.

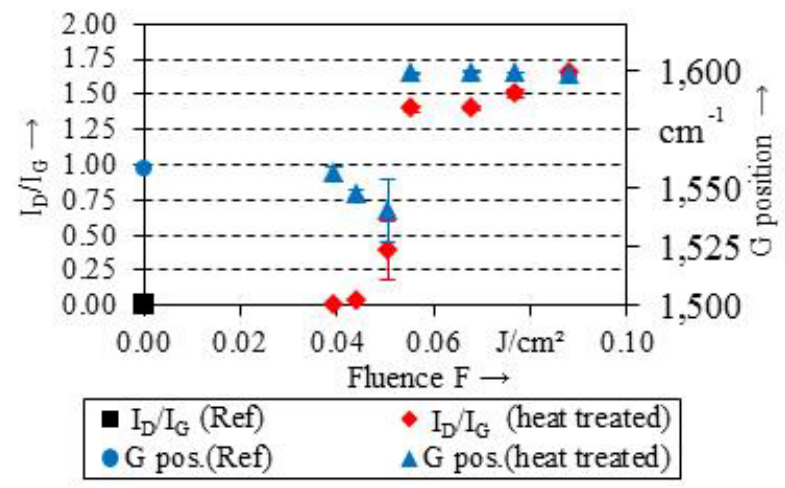

Fig. $7 \mathrm{I}_{\mathrm{D}} / \mathrm{I}_{\mathrm{G}}$-ratio and $\mathrm{G}$ peak position for the reference (Ref) and the ta-C coating heat treated with certain fluences ( $f_{P}=500 \mathrm{kHz}, w=47.5 \mu \mathrm{m}, p_{x}=0.3 \mu \mathrm{m}, p_{y}=15 \mu \mathrm{m}$, $S=1 ; n=3)$

At fluences of about $0.045 \mathrm{~J} / \mathrm{cm}^{2}$ the $I_{D} / I_{G}$-ratio slightly increases and $G$ position increases due to the decrease of $\mathrm{sp}^{3}$ hybridization which is named "hysteresis" by FERRARI and ROBERTSON [25]. For fluences higher than $0.055 \mathrm{~J} / \mathrm{cm}^{2}$ $I_{D} / I_{G}$-ratio and $\mathrm{G}$ position significantly increase due to formation of nanocrystalline (nc)-graphite. The spectra of the heat treated samples show splitting of D- and G-peak as well as increasing D-peak which signalizes clustering of $\mathrm{sp}^{2}$-phases into fairly ordered rings referred to as graphitization of the ta-C coating.

The onset of graphitization, which is characterized by $I_{D} / I_{G}$-ratios of about 0.5 , can be associated with the excess of a local surface temperature of about $1,250^{\circ} \mathrm{C}$ (Fig. 8b). This temperature threshold coincides well with the results of FERRARI ET AL. who stated that the content of $\mathrm{sp}^{2}$ phase is high enough for clustering of $\mathrm{sp}^{2}$ phases and thus, the formation of graphitic material [26]. The results of simulated surface temperature will be compared with measured data of accumulated heat in future investigations. The existence of graphitic material is visualized by the bright regions of the line-shaped structure in Fig. 8a. At the corresponding fluence of $0.055 \mathrm{~J} / \mathrm{cm}^{2}$ remarkable hardness decrease was ascertained (Fig. 7) due to thermally induced intrinsic compressive stress reduction in the ta- $\mathrm{C}$ coating. The slightly increased $I_{D} / I_{G}$-ratio for $F<0.055 \mathrm{~J} / \mathrm{cm}^{2}$ signalizes only slight $\mathrm{sp}^{2}$ phase increase [26] which does not lead to significant modification of mechanical properties. Correspondingly, structural and mechanical properties of ta-C coatings can be adjusted by picosecond laser based heat treatment whereas the surface topography in the micrometer range remains constant.

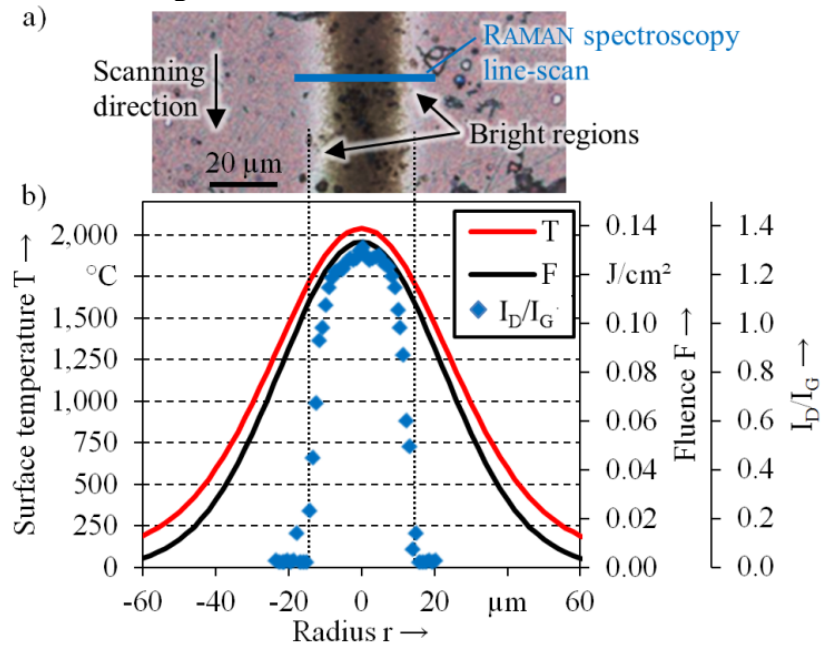

Fig. 8 a) Top view of line-shaped heat treated surface and b) calculated surface temperature $T$ at pulse $N=200$ as well as fluence $F$ and $I_{D} / I_{G}$-ratio depending on the radius $\left(f_{P}=500 \mathrm{kHz}, w=45 \mu \mathrm{m}, E_{P}=4.3 \mu \mathrm{J}, p_{x}=0.3 \mu \mathrm{m}\right.$, $S=6)$

\subsection{Laser based structuring with $1064 \mathrm{~nm}$ and $355 \mathrm{~nm}$}

Applying laser processing parameters to generate microstructures the appropriate wavelength has to be selected, too. For this purpose, the properties of $0.6 \mu \mathrm{m}$ deep structures were evaluated and compared. At $1064 \mathrm{~nm}$ spalling of the complete ta-C layer leads to comparatively steep edges which are characterized by the chamfer angle $\beta=11.24 \pm 0.02^{\circ}$ (Fig. 9a,b). The variation of the position of the structure edges amounts to $\pm 1.15 \mu \mathrm{m}$ although sufficient pulse overlap towards the scanning direction of $78 \%$ was applied. This deviation of the toothed edges corresponds to $0.6 \%$ of the structure width of $200 \mu \mathrm{m}$. Fur- 
thermore, the structure bottom is characterized by the uncovered adhesive chrome layer (Fig. 9c). Compared with this, the structure depth can be continuously adjusted applying $\lambda_{2}=355 \mathrm{~nm}$. Therefore, the structure bottom can remain covered by carbon (Fig. 9d). However, the excessive energy input for material ablation leads to significant increase of $I_{D} / I_{G}$-ratio and $\mathrm{sp}^{2}$ phase content due to residual heat (Fig. 9e). Additionally, the edge steepness can be continuously adjusted, too. The chamfer angle amounts to $1.36 \pm 0.08^{\circ}$ (Fig. 9b). The position of the structure edges varies about $\pm 0.38 \mu \mathrm{m}$. Thus, depending on the requirements on laser structuring the laser wavelength should be selected thoughtfully. If in-layer structuring with closed carbon coating is necessary, $\lambda=355 \mathrm{~nm}$ will be applied. Otherwise $\lambda=1064 \mathrm{~nm}$ can be applied to benefit from high ablation efficiency and productivity.
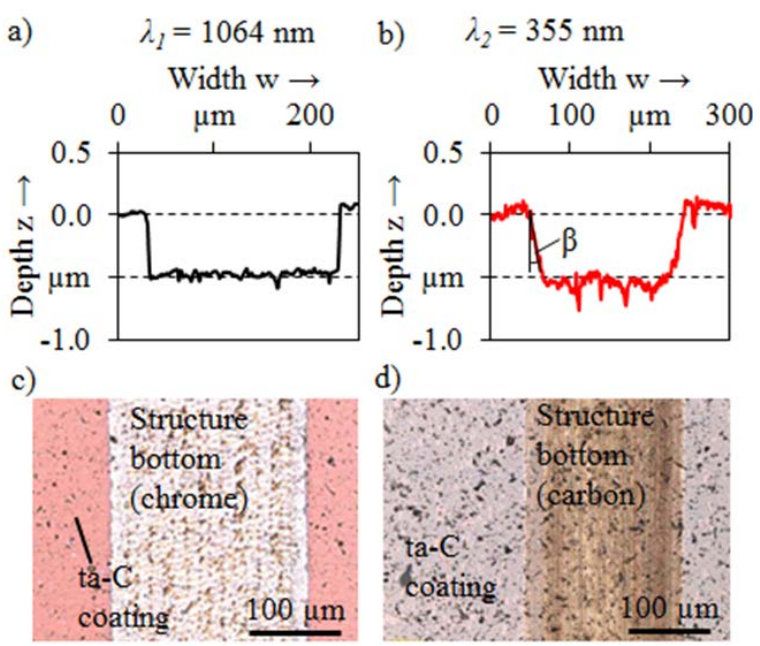

d)
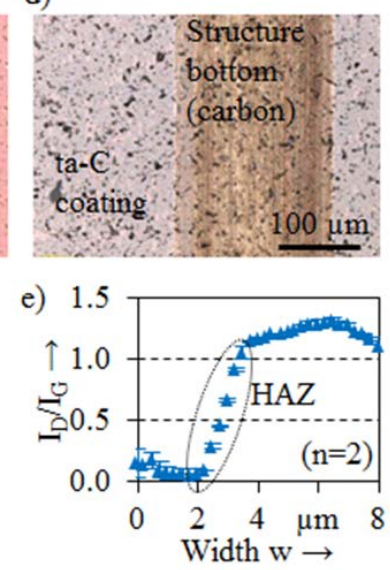

Fig. 9 Cross section (a, b), top view (c, d) and $I_{D} / I_{G}$-ratio at the edge (e) of structures generated with 1064 (parameter set A.1) and $355 \mathrm{~nm}$ (parameter set B), respectively

\subsection{Tribological behavior of heat treated ta- $C$ coatings}

Finally, the tribological behavior of the laser based surface modifications under lubricant-free conditions is analyzed in the following. Therefore, the friction coefficient and the surface conditions before and after the strip drawing test are compared. For investigation purposes, two levels of graphitization of ta-C coated surfaces were generated by applying the parameters given in chapter 3.3. The fluences were set to $F_{1}=0.070 \mathrm{~J} / \mathrm{cm}^{2} \quad$ (G1) and $F_{2}=0.075 \mathrm{~J} / \mathrm{cm}^{2}$ (G2), respectively, to realize different degrees of $\mathrm{sp}^{2}$ hybridization.

The changes of friction coefficient $\mu$ are shown in Fig. 10 . The reference ta-C coating is characterized by a friction coefficient of 0.15 in contact with DC04 sheet whereas for the graphitized friction jaws approximately 6 to $9 \%$ higher friction was measured (Fig. 10a). The lower degree of graphitization (G1) reveals only a slightly lower friction coefficient than level G2.
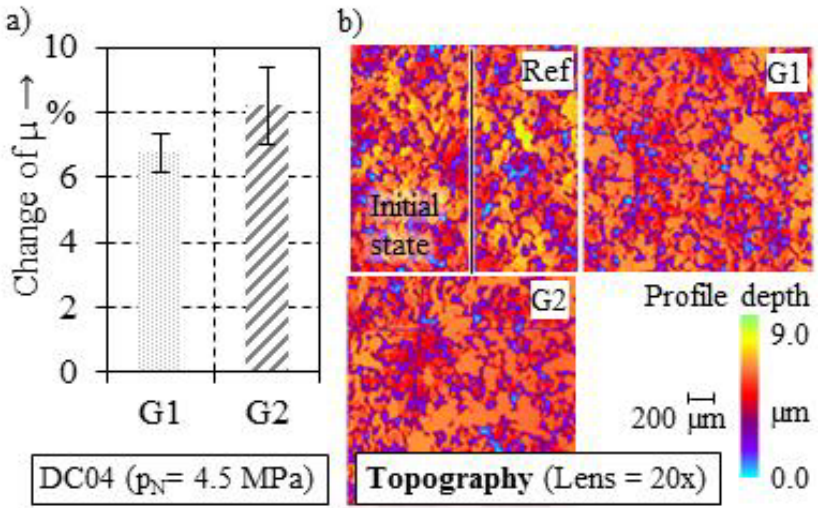

Fig. 10 a) Change of friction coefficient $\mu$ for graphitized ta-C coatings in contact with DC04 compared to non-treated reference (Ref) and b) sheet topography after strip drawing tests

Higher shear stresses between atomic layers of the hydrogen-free top coating layer and the sheet material can cause friction increase due to graphitization in lubricantfree tribological systems [27]. Furthermore, the elastic indentation modulus $E_{I T}$ (Fig. 7) decreases. This leads to reduced resistance towards elastic deformation which causes an increased flattening of the sheets (Fig. 10b) due to an increased real contact area. The characterization of the sheet surfaces revealed only a slight smoothing of the DC04 sheets for the reference test whereas for the graphitized sets G1 and G2 a smoothing of DC04 is observed.

\subsection{Tribological behavior of structured ta-C coatings}

An additional approach to adjust the tribological conditions is provided by microstructures. Therefore, the effect of microstructures in the ta- $C$ coated surface was analyzed. For this purpose, rectangular and line-shaped structures were generated in ta- $C$ coated friction jaws. The friction coefficient was determined before laser processing and afterwards to calculate a related friction coefficient. This procedure is necessary due to slight deviations of friction between different friction jaws. In contact with the zinccoated DC04 different degrees of coverage of rectangular structures were tested. The coverages $20 \%$ (R20d) and $35 \%$ (R35d) reduce the friction due to the reduced real contact area (Fig. 11).

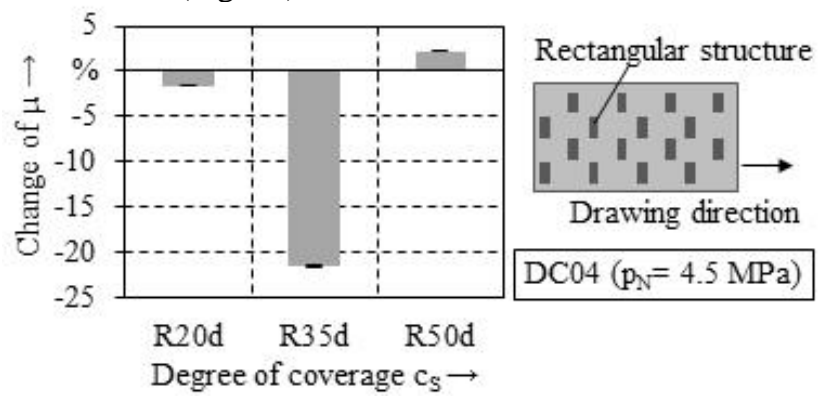

Fig. 11 Changes of friction coefficient $\mu$ due to microstructures in ta-C coated tool surfaces in contact with DC04

Consequently, the locally increased contact pressure induces smoothing of the sheet surface which leads to less interlocking asperities. However, friction increases above the value of the non-structured reference for jaws with cov- 
erage of $50 \%$ (R50d). This friction increase might be caused by a larger amount of asperities which are sheared at the edges of the structures. Thus, the positive effect of reduced real contact area with less contacting asperities is overcompensated.

In contact with AA5182 the friction jaws with coverage of $35 \%$ (R35d) revealed $200 \%$ higher friction compared to the non-structured reference. Therefore, for sheet material AA5182 structure with different shape and a depth of $0.6 \mu \mathrm{m}$ were tested to prevent exposure of the steel substrate with its high adhesion tendency towards aluminum. However, the same frictional behavior was observed for flat rectangular structures (R35f) (Fig. 12). Flat line-shaped structures with the same depth and degree of coverage (L35f) revealed even higher related friction of $315 \%$ although the interlocking of asperities at the edges of structures perpendicular towards drawing direction should be reduced. This increase might be induced by the asperities of the sheet surface which are in the areas of the line-shape structure and contact the bottom of the structures. These asperities are not smoothed at the ta- $C$ surface and could increase resistance towards relative movement due to its adhesion tendency towards the still exposed adhesive chrome layer. To prevent adhesion to any metallic material a ta-C coating with a thickness of $1.3 \mu \mathrm{m}$ was deposited. Flat line-shaped structures in the thick ta-C layer (L35f*) led to $10 \%$ higher friction compared to the reference. This result might be explained by the completely covered chrome layer at the structure bottom. The small amount of aluminum particles which still adheres and accumulates at the structure bottom could be induced by the increased degree of $\mathrm{sp}^{2}$ hybridization (chapter 3.4 ) and its slightly higher adhesion tendency compared to the non-treated ta-C reference. On the basis of these results direct contact with chrome and steel has to be avoided to reduce friction and adhesion for AA5182. Further details about the tribological behavior of laser modified tool surfaces for dry deep drawing are discussed in Ref. [28].

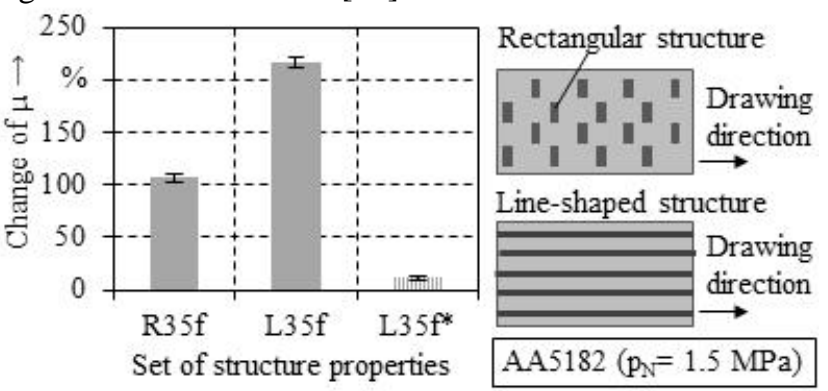

Fig. 12 Changes of friction coefficient $\mu$ due to $0.6 \mu \mathrm{m}$ deep microstructures in ta-C coated tool surfaces in contact with AA5182

\section{Conclusion and outlook}

The possibilities and characteristics of picosecond laser based tailored modification of ta-C coatings were investigated. Processing by $\lambda=355 \mathrm{~nm}$ enables highest potential to adjust topographical, mechanical and structural properties. This is reasoned by the optical properties of this amorphous carbon coating. Maximum width of a process window in terms of fluence range for laser based heat treat- ment to reduce hardness and increase $\mathrm{sp}^{2}$ hybridization can be achieved by applying minimum pulse pitch and maximum pulse frequency. Thus, Vickers hardness can be decreased up to $70 \%$ compared to the non-treated ta-C reference. Most efficient structuring can be realized by the application of $\lambda=1064 \mathrm{~nm}$ due to local spalling of the whole ta-C layer. In contrast to this, accuracy of material ablation is higher with $355 \mathrm{~nm}$. However, residual heat leads to remarkable graphitization of the generated structure bottom.

Regarding the effect of graphitization on the behavior of lubricant-free tribological systems friction is increased with higher $\mathrm{sp}^{2}$ hybridization in contact with zinc-coated DC04. This friction growth is caused by decreased elastic indentation modulus of heat treated ta-C surface. Thus, friction can be locally adjusted by heat treatment without the change of surface topography and accompanying risk of abrasion. Beyond that, laser structuring of the coated surfaces leads to either friction decrease or increase depending on the area coverage and orientation of the microstructures. Additionally, the microstructures are able to trap the wear particles. Therefore, friction was adapted in a range of $80 \%$ to $110 \%$ compared to the reference for DC04. The real contact area, the amount of contacting roughness asperities and shearing of asperities on the structure edges are influenced. The predominant tribological mechanism is affected by the degree of coverage. For AA5182 friction increased significantly up to $220 \%$ due to high adhesion tendency of AA5182 towards the structure bottom which consists of non-covered chrome. Structures with a closed ta-C layer enable friction coefficient marginally higher than the reference. As a consequence, properties of desired modifications as well as laser processing parameters have to be chosen wisely to realize tailored tribological conditions in dry forming.

\section{Acknowledgments}

The authors gratefully acknowledge funding of the SPP1676 project SCHM 2115/49-1, ME 2043/50-1 and TR 1043/5-1 with the title "Lubrication free forming with tailored tribological conditions" and the funding of the Erlangen Graduate School in Advanced Optical Technologies (SAOT) by the German National Science Foundation (DFG) in the framework of the excellence initiative. Furthermore, the authors thank H-O-T GmbH \& Co. KG for coating deposition.

\section{References}

[1] F. Vollertsen, F. Schmidt: Dry Metal Forming: Definition, Chances and Challenges. International Journal of Precision Engineering and Manufacturing - Green Technology, 1, (2014) 59.

[2] M. Merklein, M. Schmidt, S. Tremmel, K. Andreas, T. Häfner, R. Zhao, J. Steiner: Tailored modifications of amorphous carbon based coatings for dry deep drawing. Dry Metal Forming Open Access Journal, 2, (2016) 25.

[3] Association of German Engineers (VDI): VDI Guideline 2840. Beuth (2012).

[4] Y. Lifshitz, G.D. Lempert, E. Grossman: Substantiation of subplantation model for diamondlike film growth by atomic force microscopy. Phys. Rev. Lett., 72, (1994) 2753. 
[5] T. Roch, V. Weihnacht, H.-J. Scheibe, A. Roch, A. Lasagni: Direct Laser Interference Patterning of tetrahedral amorphous carbon films for tribological applications: Diam. \& Rel. Mat., 33, (2013) 20.

[6] T. Roch, A. Lasagni, E. Beyer: Nanosecond UV laser graphitization and delamination of thin tetrahedral amorphous carbon films with different $\mathrm{sp}^{3} / \mathrm{sp}^{2}$ content: Thin Solid Films, 519, (2011) 3756.

[7] T. Nakamiya, S. Aoqui, K. Ebihara: Experimental and numerical study on pulsed-laser annealing process of diamond-like carbon thin films. Diam. Rel. Mat., 10, (2001) 905.

[8] T. Stucky, U. Baier, C.-F. Meyer, H.-J. Scheibe, B. Schultrich: Großflächenbeschichtung mit superhartem Kohlenstoff. Vakuum in Forschung und Praxis, 6, (2003).

[9] German Institute for Standardization (DIN): DIN EN ISO 25178. Berlin, Beuth (2011).

[10] A.C. Ferrari, J. Robertson: Raman spectroscopy of amorphous, nanostructured, diamond like carbon, and nanodiamond. Philosophical Transactions of the Royal Society of London A, 362, (2004) 2477.

[11] S. Prawer, K.W. Nugent, Y. Lifshitz, G.D. Lempert, E. Grossman, J. Kulik, I. Avigal, R. Kalish: Systematic variation of the Raman spectra of DLC films as a function of sp2:sp3 composition. Diamond and Related Materials, 5, (1996) 438.

[12] German Institute for Standardization (DIN): DIN EN ISO 14577. Berlin, Beuth (2003).

[13] B.K. Tay, X. Shi, E. Liu, H.S. Tan, L.K.Cheah, J. Shi, E.C. Lim, H.Y. Lee: Tribological and optical properties of hydrogen-free Amorphous Carbon Films with Varying $\mathrm{sp}^{3} / \mathrm{sp}^{2}$ Composition. Surface and Interface Analysis, 28, (1999) 226.

[14]E.T. Arakawa, S.M. Dolfini, J.C. Ashley, M.W. Williams: Arc-evaporated carbon films: Optical properties and electron mean free paths. Phys. Rev. B, 31, (1985) 8097.

[15] R. Pastorelli, A.C. Ferrari, M.G. Beghi, C.E. Bottani, J. Robertson: Elastic constants of ultrathin diamond-like carbon films: Diam. \& Rel. Mat., 9, (2000), 825.

[16]F. Di et al: Preparation and Characteristics of Nanoscale Diamond-Like Carbon Films for Resistive Memory Applications. Cin. Phys. Lett., 27, (2010) 098102.

[17] M. Shamsa, W. L. Liu, A. A. Balandin, C. Casiraghi, W. I. Milne, A. C. Ferrari: Thermal conductivity of diamond-like carbon films. Appl. Phys. Lett. 89, (2006), 161921.

[18]G. Radons, H. Wirth.: "Debyesche Theorie der Wärmekapazität: Zur Erläuterung des DebyeModells.“ In: „Lexikon der Physik“ ed. by W. Greulich (Spektrum Akademischer Verlag, Heidelberg, 1998).

[19] C. Ullrich, T. Bodmer: "VDI-Wärmeatlas” ed. by Association of German Engineers (VDI) (Springer, Berlin, Heidelberg, 2013) p.629.

[20] Böhler-Uddeholm: “AISI D2 Cold work tool steel”, data sheet, 1, (2013).

[21] J.M. Liu: Simple Technique for Measurements of Pulsed Gaussian-beam Spot Sizes. Opt. Lett., 7, (1982) 196.
[22] L.M. Machado, R.E. Samad, W. de Rossi, N.D. Vieira Junior: D-Scan measurement of ablation threshold incubation effects for ultrashort laser pulses. Opt. Expr., 20, (2012), 4114.

[23] T. Häfner, J. Heberle, M. Dobler, M. Schmidt: Influences on Incubation in ps Laser Micro-machining of Steel Alloys. J. of Laser Appl., 28, (2016) 0226051.

[24] A.C. Ferrari; J. Robertson: Resonant Raman spectroscopy of disordered, amorphous, and diamond-like carbon. Phys. Rev. B, 64, (2001), 075414.

[25] A.C. Ferrari, J. Robertson: Interpretation of Raman spectra of disordered and amorphous carbon. Phys. Rev. B, 61, (2000), 14095.

[26] A.C. Ferrari, et al.: Stress reduction and bond stability during thermal annealing of tetrahedral amorphous carbon. J. Appl. Phys., 85, (1999) 7191.

[27]H. Ronkainen, K. Holmberg: "Environmental and Thermal Effects on the Tribological Performance of DLC Coatings” ed by C. Donnet, A. Erdemir (Springer Science, New York, 2008) p.155.

[28] J. Tenner, R. Zhao, S. Tremmel, T. Häfner, M. Schmidt, M. Merklein: Tribological Behavior of Carbon Based Coatings Adapted to Lubricant-free Forming Conditions: Proc. Int. Symp. on Green Manufacturing and Applications, Gyeongju, (2017) (accepted)

(Received: June 4, 2017, Accepted: August 24, 2017) 